\title{
The accuracy of chromosomal microarray testing for identification of embryonic mosaicism in human blastocysts
}

Veronica Novik*, Emily B Moulton, Michael E Sisson, Shagun L Shrestha, Khoa D Tran, Harvey J Stern, Brian D Mariani and Wayne S Stanley

\begin{abstract}
Background: Most previous studies of chromosomal mosaicism in IVF embryos were performed by fluorescence in situ hybridization (FISH) methods. While there are reports implicating chromosome aneuploidy in implantation failure following transfer and pregnancy loss by spontaneous miscarriage, the significance of mosaicism for the developmental potential of growing embryos is unknown. However, the low prevalence of chromosomal mosaicism in chorionic villus sampling and amniotic fluid specimens suggests the presence of selection against mosaic embryos for implantation and early pregnancy. The absence of evidence for selective allocation of abnormal cells to the trophectoderm (TE) of mosaic blastocysts permits these cells to be a good proxy for embryonic mosaicism detection by chromosomal microarrays (CMA). The purpose of this study was to establish the limits of detection and the prevalence of chromosome mosaicism in day 5/6 human embryos using CMA with TE biopsies.

Results: From reconstitution experiments we established $\log _{2}$ ratio thresholds for mosaicism detection. These studies indicated that chromosomal mosaicism at levels as low as between 25-37\% can be consistently identified. Follow-up studies by FISH on non-transferred abnormal embryos confirmed the diagnostic accuracy of CMA testing. The number of cells in a TE biopsy can influence mosaicism detection.

Conclusions: Chromosomal microarrays can detect mosaicism in TE biopsies when present at levels as low as between $25-37 \%$ and the prevalence of day 5/6 blastocysts which were mosaic and had no other abnormalities reached 15\% among a cohort of 551 embryos examined. Validated protocols for establishing detection thresholds for mosaicism are important to reduce the likelihood of transferring abnormal embryos.
\end{abstract}

\section{Background}

Chromosomal mosaicism has been associated with human morbidity but the phenotypic manifestations are dependent on mosaicism level, the chromosome involved, and tissue distribution. An accurate assessment of the prevalence of mosaicism in the newborn population is uncertain because of ascertainment bias toward abnormal clinical presentations. However, in prenatal specimens large studies indicate that mosaicism is found in 0.20 $0.25 \%$ of amniotic fluid $[1,2]$ and $0.8-2 \%$ of chorionic villus samples [3-6]. Chromosomal mosaicism is significantly higher in IVF created embryos than in other prenatal specimens. In a recent literature review and meta-analysis

\footnotetext{
* Correspondence: vnovik@givf.com

Genetics and IVF Institute, Fairfax, VA, USA
}

of human preimplantation embryo chromosomal mosaicism, van Echten-Arends et al. [7] reported that mosaicism is the most common chromosome constitution in spare IVF embryos and was found in $73 \%$ of all embryos. Of these embryos, $67 \%$ were at cleavage stage and most of the studies used FISH to determine chromosome constitution as well as spare embryos that were deemed unsuitable for transfer. Using chromosomal microarrays with cleavage stage embryos, Mertzamidou et al. [8] also found $71 \%$ of embryos to be mosaic. Most previous studies assessed mosaicism at cleavage stage from spare or arrested embryos. There is currently little data on chromosomal mosaicism derived from progressing day 5/6 hatching blastocysts.

Chromosomal microarrays have replaced FISH and comparative genomic hybridization (CGH) based methods for

\section{Biomed Central}

(c) 2014 Novik et al.; licensee BioMed Central Ltd. This is an Open Access article distributed under the terms of the Creative Commons Attribution License (http://creativecommons.org/licenses/by/2.0), which permits unrestricted use, distribution, and reproduction in any medium, provided the original work is properly credited. The Creative Commons Public Domain Dedication waiver (http://creativecommons.org/publicdomain/zero/1.0/) applies to the data made available in this article, unless otherwise stated. 
assessing aneuploidy in developing embryos $[9,10]$ and aneuploidy has been implicated in implantation failure and pregnancy loss by spontaneous miscarriage following IVF embryo transfer $[11,12]$. The relatively low levels of mosaicism reported in prenatal specimens and the decreasing frequency of mosaicism with increased gestational age suggests the presence of selective pressure against embryos with high levels of mosaicism for ongoing pregnancy. Although mosaicism has been reported in embryos using CMAs with TE biopsies [13,14], the effects of this mosaicism on embryonic development, implantation, and pregnancy outcome is currently unknown and the degree to which a CMA result from a small sample of embryonic cells at day $5 / 6$ accurately reflects embryonic mosaicism levels has not been adequately described. We report here on the limits of mosaicism detection using cell mixing experiments, the accuracy of mosaicism detection from FISH follow-up studies, and estimate the prevalence of mosaicism at the blastocyst stage of development.

\section{Results}

\section{Reconstitution experiments and establishment of $\log _{2}$} ratios to detect chromosomal mosaicism

To mimic chromosomal gains and losses, mixing experiments with different ratios of amplified DNA from aneuploid and euploid cell lines as well as clinical samples were used as described in the Methods section. The sample size of five cells was chosen for SurePlex amplification because it is similar to the size of an average trophectoderm biopsy. Individual $\log _{2}$ ratios and average $\log _{2}$ ratios were determined for trisomy 13 and trisomy 21 samples at different levels of mosaicism (Additional file 1) and likewise for monosomy 6 and monosomy 22 (Additional file 2). A clear and definitive visualization of all hybridization signals above or below the $0 \log _{2}$ ratio line, the first indicative sign of chromosomal mosaicism, was detected at levels as low as between $25-37.5 \%$ (a $\log _{2}$ ratio of +0.135 ) for gains and at $37-50 \%$ (an average $\log _{2}$ ratio of -0.153) for losses. The Additional files 3 and 4 provide illustrations of chromosomal aneuploidy at 0 , $25,37.5,50,75$ and $100 \%$ for a trisomy $(+13)$ and a monosomy (-6), respectively. Also of note is that the visual detection of chromosomal mosaicism is largely dependent on the quality of the starting material. Trophectoderm biopsy samples from degenerating embryos usually lead to a noisy pattern of array hybridization profiles which could obscure the detection of mosaicism. We blind-tested twelve mosaic and non-mosaic samples to further confirm our criteria - all samples were identified correctly (Additional file 5).

\section{FISH follow-up analysis of research embryos}

The diagnostic accuracy of our CMA testing was assessed by FISH follow-up with non-transferred abnormal embryos. Only chromosomes identified to be aneuploid (non-mosaic or mosaic) by CMA were targeted by FISH probes. The results for embryos diagnosed as having at least one non-mosaic chromosome aneuploidy are shown in Table 1. For scoring purposes, an embryo FISH result was considered abnormal and concordant with the TE result when nuclei had the same abnormality identified by CMA or a complementary abnormality (e.g. +21 or -21 ) as could be found in mosaic embryos. Of the 26 embryos identified to be non-mosaic by CMA, FISH follow-up confirmed abnormalities in all of them (100\%) but $50 \%$ of the embryos in this group were mosaic for at least one chromosome by FISH. The results for embryos diagnosed by CMA as having at least one mosaic chromosome aneuploidy are shown in Table 2. Of the 21 embryos in this group, 20 embryos were abnormal (95\%). Nineteen embryos (90\%) were confirmed as having at least one mosaic chromosome and one embryo was found by FISH to be non-mosaic aneuploid. For embryos with two or more mosaic chromosomes, FISH demonstrated that all these embryos contained some non-mosaic cells and thus did not represent aneuploid-aneuploid mosaic embryos. These results confirm the diagnostic accuracy of CMA testing for both non-mosaic and mosaic embryo aneuploidy categories and thus permit a realistic estimation of the prevalence of euploidy and aneuploidy in a larger cohort of day $5 / 6$ blastocysts.

\section{Aneuploidy and mosaicism in day $5 / 6$ embryos}

From CMA analysis of 551 blastocysts obtained during IVF cycles at our clinic (Table 3), 52.5\% were euploid and $47.5 \%$ were aneuploid. In the chromosomally aneuploid group, $40.5 \%$ were non-mosaic, $31.6 \%$ displayed chromosome mosaicism only (using the established criteria for chromosomal mosaicism described above), and the remaining abnormal embryos (27.9\%) displayed both mosaic and non-mosaic forms of aneuploidy. Given that our follow-up studies indicated that about one half of CMA diagnosed non-mosaic embryos were mosaic, then the true number of embryos that are non-mosaic aneuploid is likely smaller and the mosaic only and mixed non-mosaic/mosaic aneuploid subgroups are likely larger. When this embryo cohort was analyzed by maternal age (Table 4), the percent of euploid embryos decreased with increased maternal age. There was also a direct relationship between increased maternal age and percent of non-mosaic aneuploid embryos; the ratio of non-mosaic aneuploid embryos to euploid embryos in the 25-34 age group was 0.24 but 1.67 in the age 40-42 group. The ratio of mosaic only embryos to euploid embryos was 0.26 in the youngest age group and 0.33 in the oldest group. 
Table 1 Follow-up FISH data for embryos diagnosed with non-mosaic aneuploidy by CMA

\begin{tabular}{|c|c|c|c|c|c|c|c|}
\hline $\begin{array}{l}\text { Specimen } \\
\text { designation }\end{array}$ & TE cell \# & $\begin{array}{c}\text { CMA } \\
\text { aneuploidy }^{\mathrm{a}}\end{array}$ & $\log _{2}$ ratio & $\begin{array}{l}\text { Abnormal nuclei } \\
\text { by FISH }(\%)^{b}\end{array}$ & Non-mosaic ${ }^{c}$ & Mosaic $^{d}$ & $\begin{array}{l}\text { Embryo confirmed } \\
\text { abnormal }^{\mathrm{e}}\end{array}$ \\
\hline \multirow[t]{2}{*}{$1 \mathrm{NM}$} & 6 & $-X$ & -0.501 & 100 & Yes & No & Yes \\
\hline & & -13 & -0.461 & 100 & Yes & No & \\
\hline $2 \mathrm{NM}$ & 3 & +15 & 0.284 & 84 & No & Yes & Yes \\
\hline \multirow[t]{3}{*}{$3 \mathrm{NM}$} & 2 & $-x$ & -0.721 & 37 & No & Yes & Yes \\
\hline & & $+Y$ & 0.440 & 47 & No & Yes & \\
\hline & & +21 & 0.369 & 47 & No & Yes & \\
\hline \multirow[t]{2}{*}{$4 \mathrm{NM}$} & 2 & -13 & -0.409 & 21 & No & Yes & Yes \\
\hline & & -18 & -0.446 & 47 & No & Yes & \\
\hline $5 \mathrm{NM}$ & 6 & -22 & -0.430 & 100 & Yes & No & Yes \\
\hline $6 \mathrm{NM}$ & 5 & -6 & -0.487 & 100 & Yes & No & Yes \\
\hline $7 \mathrm{NM}$ & 7 & -14 & -0.374 & 94 & Yes & No & Yes \\
\hline $8 \mathrm{NM}$ & 7 & +15 & 0.300 & 94 & Yes & No & Yes \\
\hline \multirow[t]{2}{*}{$9 \mathrm{NM}$} & 2 & $-Y$ & -0.237 & 0 & No & No & Yes \\
\hline & & +21 & 0.465 & 92 & Yes & No & \\
\hline $10 \mathrm{NM}$ & 5 & +15 & 0.317 & 97 & Yes & No & Yes \\
\hline $11 \mathrm{NM}$ & 2 & -15 & -0.508 & 74 & No & Yes & Yes \\
\hline $12 \mathrm{NM}$ & 4 & -19 & -0.382 & 91 & Yes & No & Yes \\
\hline \multirow[t]{3}{*}{$13 \mathrm{NM}$} & 3 & +5 & 0.294 & 80 & No & Yes & Yes \\
\hline & & +14 & 0.249 & 83 & No & Yes & \\
\hline & & -18 & -0.529 & 100 & Yes & No & \\
\hline $14 \mathrm{NM}$ & 3 & -14 & -0.555 & 73 & No & Yes & Yes \\
\hline $15 \mathrm{NM}$ & 1 & -22 & -0.395 & 94 & Yes & No & Yes \\
\hline \multirow[t]{2}{*}{$16 \mathrm{NM}$} & 4 & -8 & -0.642 & 97 & Yes & No & Yes \\
\hline & & +11 & 0.336 & 97 & Yes & No & \\
\hline $17 \mathrm{NM}$ & 3 & -21 & -0.432 & 73 & No & Yes & Yes \\
\hline $18 \mathrm{NM}$ & 2 & +9 & 0.375 & 94 & Yes & No & Yes \\
\hline $19 \mathrm{NM}$ & 5 & +16 & 0.253 & 86 & No & Yes & Yes \\
\hline $20 \mathrm{NM}$ & 4 & -22 & -0.407 & 87 & No & Yes & Yes \\
\hline \multirow[t]{2}{*}{$21 \mathrm{NM}$} & 3 & -4 & -0.440 & 12 & No & Yes & Yes \\
\hline & & -10 & -1.540 & 0 & No & No & \\
\hline \multirow[t]{2}{*}{$22 \mathrm{NM}$} & $1-2$ & $-X$ & -0.485 & 87 & No & Yes & Yes \\
\hline & & +13 & 0.398 & 71 & No & Yes & \\
\hline \multirow[t]{2}{*}{$23 \mathrm{NM}$} & 4 & +8 & 0.288 & 94 & Yes & No & Yes \\
\hline & & +22 & 0.268 & 97 & Yes & No & \\
\hline $24 \mathrm{NM}$ & 8 & -19 & -0.417 & 80 & No & Yes & Yes \\
\hline $25 \mathrm{NM}$ & 3 & +22 & +0.256 & 86 & No & Yes & Yes \\
\hline $26 \mathrm{NM}$ & 3 & -10 & -0.561 & 94 & Yes & No & Yes \\
\hline
\end{tabular}

all chromosomes listed as abnormal in the embryos of this table were identified by BlueFuse software to be aneuploid.

${ }^{\mathrm{b}} \mathrm{A}$ nucleus is considered abnormal by FISH if there is a loss or gain of the chromosome identified by CMA to be aneuploid.

${ }^{C} \mathrm{~A}$ non-mosaic CMA result is considered concordant with FISH if $\geq 90 \%$ of follow-up embryo cells have a FISH signal pattern consistent with the CMA data.

${ }^{d}$ A mosaic CMA result is considered concordant with FISH if $\geq 10 \%$ and $<90 \%$ of follow-up embryo cells have a FISH signal pattern consistent with the CMA data.

${ }^{\mathrm{e}} \mathrm{An}$ embryo follow-up result is considered abnormal and concordant with an abnormal CMA result if at least one chromosome in an embryo identified by CMA as abnormal is confirmed by FISH.

\section{Discussion}

Chromosomal microarrays have documented aneuploidy and mosaicism to be common at the blastocyst stage of development $[7,13-16]$. While arrays can confidently identify non-mosaic aneuploidy, there is less certainty regarding the technical limits of detection of mosaicism especially when the biopsy cell number is limited and whole genome amplification is required. Because microarray data is dependent on array platform, the quality of DNA and software algorithms, it is important that 
Table 2 Follow-up FISH data for embryos diagnosed with mosaic aneuploidy by CMA

\begin{tabular}{|c|c|c|c|c|c|c|c|}
\hline $\begin{array}{l}\text { Specimen } \\
\text { designation }\end{array}$ & TE cell \# & $\begin{array}{c}\text { CMA } \\
\text { aneuploidy }^{\mathrm{a}}\end{array}$ & $\log _{2}$ ratio & $\begin{array}{l}\text { Abnormal nuclei } \\
\text { by FISH } \%^{\text {b }}\end{array}$ & Non-mosaic ${ }^{c}$ & Mosaic $^{d}$ & $\begin{array}{l}\text { Embryo confirmed } \\
\text { abnormal }^{\mathrm{e}}\end{array}$ \\
\hline $1 \mathrm{M}$ & 2 & +14 & 0.193 & 97 & Yes & No & Yes \\
\hline $2 \mathrm{M}$ & 4 & +8 & 0.206 & 53 & No & Yes & Yes \\
\hline $3 M$ & 3 & -1 & -0.155 & 10 & No & Yes & Yes \\
\hline $4 \mathrm{M}$ & 5 & -4 & -0.235 & 21 & No & Yes & Yes \\
\hline $5 \mathrm{M}$ & 4 & -19 & -0.173 & 20 & No & Yes & Yes \\
\hline $6 \mathrm{M}$ & 4 & -4 & -0.154 & 10 & No & Yes & Yes \\
\hline \multirow[t]{2}{*}{$7 \mathrm{M}$} & 4 & +5 & 0.220 & 27 & No & Yes & Yes \\
\hline & & +10 & 0.133 & 6 & No & No & \\
\hline $8 \mathrm{M}$ & 4 & -8 & -0.203 & 20 & No & Yes & Yes \\
\hline $9 M$ & 3 & -14 & -0.178 & 12 & No & Yes & Yes \\
\hline $10 \mathrm{M}$ & 2 & -9 & -0.183 & 7 & No & No & No \\
\hline \multirow[t]{2}{*}{$11 \mathrm{M}$} & 4 & +15 & 0.230 & 63 & No & Yes & Yes \\
\hline & & +17 & 0.164 & 20 & No & Yes & \\
\hline \multirow[t]{2}{*}{$12 \mathrm{M}$} & 11 & -8 & -0.280 & 19 & No & Yes & Yes \\
\hline & & -11 & -0.141 & 28 & No & Yes & \\
\hline $13 \mathrm{M}$ & 7 & -8 & -0.230 & 71 & No & Yes & Yes \\
\hline \multirow[t]{2}{*}{$14 \mathrm{M}$} & 4 & +2 & 0.211 & 40 & No & Yes & Yes \\
\hline & & +8 & 0.181 & 26 & No & Yes & \\
\hline $15 \mathrm{M}$ & 4 & +22 & 0.136 & 16 & No & Yes & Yes \\
\hline $16 \mathrm{M}$ & 4 & +1 & 0.168 & 15 & No & Yes & Yes \\
\hline $17 \mathrm{M}$ & 4 & -18 & -0.216 & 58 & No & Yes & Yes \\
\hline \multirow[t]{2}{*}{$18 \mathrm{M}$} & 4 & -8 & -0.199 & 43 & No & Yes & Yes \\
\hline & & +16 & 0.179 & 30 & $\mathrm{No}$ & Yes & \\
\hline \multirow[t]{2}{*}{$19 \mathrm{M}$} & 2 & -3 & -0.146 & 23 & No & Yes & Yes \\
\hline & & $+X$ & 0.159 & 19 & No & Yes & \\
\hline \multirow[t]{2}{*}{$20 \mathrm{M}$} & 4 & +19 & 0.159 & 23 & No & Yes & Yes \\
\hline & & $+X$ & 0.149 & 26 & No & Yes & \\
\hline $21 \mathrm{M}$ & $3-4$ & -19 & -0.162 & 56 & No & Yes & Yes \\
\hline
\end{tabular}

${ }^{a}$ Embryos listed in this table were identified by CMA to have at least one mosaic chromosome aneuploidy.

${ }^{\mathrm{b}} \mathrm{A}$ nucleus is considered abnormal by FISH if there is a loss or gain of the chromosome identified by CMA to be aneuploid.

${ }^{\mathrm{C}} \mathrm{A}$ non-mosaic CMA result is considered concordant with FISH if $\geq 90 \%$ of follow-up embryo cells have a FISH signal pattern consistent with the CMA data.

${ }^{\mathrm{d} A}$ mosaic CMA result is considered concordant with FISH if $\geq 10 \%$ and $<90 \%$ of follow-up embryo cells have a FISH signal pattern consistent with the CMA data. ${ }^{\mathrm{e}} \mathrm{An}$ embryo follow-up result is considered abnormal and concordant with an abnormal CMA result if at least one chromosome in an embryo identified by CMA as abnormal is confirmed by FISH.

individual laboratories develop their own protocols for assessing mosaicism. This has been recommended by both the American College of Medical Genetics and the European best practices guidelines for constitutional microarray testing $[17,18]$. As a prerequisite for assessing the accuracy of arrays for the identification of embryonic mosaicism, we first determined the limits of detection of mosaicism by performing reconstitution experiments using DNA mixed from normal and aneuploid samples and analyzing CMA results for mosaicism detection using BlueGnome's 24sure array and data generated by BlueGnome's BlueFuse Multi software.

Table 3 Summary of CMA data on embryo mosaicism

\begin{tabular}{ccccc}
\hline $\begin{array}{c}\text { Total number of } \\
\text { embryos analyzed }\end{array}$ & Normal (\%) & \multicolumn{2}{c}{ Abnormal, $\mathbf{n}=\mathbf{2 6 2}(\mathbf{4 7 . 5 \% )}$} \\
\cline { 2 - 5 } & & Aneuploid $^{\mathbf{b}}$ (\%) & Aneuploid $^{\mathbf{b}}{\text { and } \text { mosaic }^{\mathbf{c}} \text { (\%) }}^{\text {Mosaic }^{\mathbf{c}} \text { (\%) }}$ \\
\hline 551 & $289 / 551(52.5)$ & $106 / 262(40.5)$ & $73 / 262(27.9)$ & $83 / 262(31.6)$ \\
\hline
\end{tabular}

${ }^{a}$ All age groups combined: the maternal age averaged across all blastocysts in cohort was 33.9. Embryos obtained from 120 PGD cycles (103 patients total). Donor eggs were used in 21 cycles; the maternal age for this group was calculated as 33 .

${ }^{b}$ Embryo was called aneuploid if at least one chromosome was identified by BlueFuse software to be aneuploid at diagnosis.

${ }^{c}$ Embryo was called mosaic if at least one chromosome had a $\log _{2}$ ratio CMA result of $\geq+0.130$ or $\leq-0.150$ at diagnosis and a homogeneous deflection of all hybridization signals. 
Table 4 Correlation between embryo euploidy/aneuploidy and maternal age

\begin{tabular}{lccccc}
\hline $\begin{array}{l}\text { Age } \\
\text { group }\end{array}$ & $\begin{array}{c}\text { Total number of } \\
\text { embryos analyzed }\end{array}$ & Normal (\%) & \multicolumn{2}{c}{ Abnormal: $\boldsymbol{n}$ varies according to the age group (\%) } \\
\cline { 4 - 6 } & 338 & $202 / 338(59.8)$ & $48 / 136(35.3)$ & Aneuploid $^{\mathbf{a}}{\text { and } \text { mosaic }^{\mathbf{b}}(\%)}^{\text {Mosaic }^{\mathbf{b}}(\%)}$ \\
\hline $25-34$ & 163 & $75 / 163(46)$ & $38 / 88(43.2)$ & $35 / 136(25.8)$ & $53 / 136(38.9)$ \\
\hline $35-39$ & 50 & $12 / 50(24)$ & $20 / 38(52.6)$ & $24 / 88(27.3)$ & $26 / 88(29.5)$ \\
\hline $40-42$ & & & $14 / 38(36.9)$ & $4 / 38(10.5)$ \\
\hline
\end{tabular}

${ }^{a}$ Embryo was called aneuploid when BlueFuse software called a chromosome(s) gain or loss.

${ }^{b}$ Embryo was called mosaic if at least one chromosome had a $\log _{2}$ ratio CMA result of $\geq+0.130$ or $\leq-0.150$ at diagnosis and a homogeneous deflection of all hybridization signals.

In our experiments we attempted to identify and characterize the approximate lowest levels of mosaicism that could be confirmed from follow-up studies as biologically real. We interpreted the presence of whole chromosome mosaicism when a clear visual deflection of all hybridization signals above or below the $\log _{2} 0$ ratio value was discernible for any chromosome. For chromosome gains, mosaicism as low as between $25-37 \%$ can be observed which corresponds to a $\log _{2}$ ratio of approximately +0.13 and for chromosome losses, mosaicism as low as between $37-50 \%$ can be observed which corresponds to a $\log _{2}$ ratio of approximately -0.15 (see Additional files 1, 2, 3 and 4). There was some variability around these cut-off levels, as expected, because BAC arrays have less reproducibility and more batch-to-batch variation when compared to oligonucleotide constructed arrays [19]. Variation in array lots and in whole genome DNA amplification with small samples can also influence $\log _{2}$ ratios. In addition, there are also likely to be minor chromosome specific $\log _{2}$ ratio differences between different individual chromosome trisomies and monosomies due to variation in the number of probes for each chromosome and their unique hybridization properties. The predicted $\log _{2}$ ratio for a chromosome gain is $+0.58\left[\log _{2}\right.$ $(3 / 2)]$ and that for a chromosome loss is $-1.0\left[\log _{2}(1 / 2)\right]$. It might be expected, then, that deflection of hybridization signals below the $\log _{2}$ ratio 0 value would be more prominent for chromosome losses compared to gains and that mosaicism for losses would be relatively easier to identify than for gains. Others have demonstrated that copy number variation $(\mathrm{CNV})$ duplications are obscured by lower levels of maternal cell contamination than for similarly sized CNV deletions [20]. In patient samples displaying both a chromosome gain(s) and loss(es), we usually find the deviation from the $\log _{2} 0$ line is greater for chromosome losses than for chromosome gains (unpublished observations). In these situations, losses and gains were determined from the same pool of amplified DNA. However, in our reconstruction experiments where we did not detect lower levels of mosaicism for chromosome losses compared to chromosome gains, these results are likely to be an experimental artifact due to the combining of different pools of amplified DNA products where there could have been minor differences in the quality of the DNA prior to whole genome amplification. The results we obtained for chromosome gains are consistent with those of Mamas et al. [21] who used the same array platform and software but analyzed DNA extracted from mixed populations of aneuploid and normal cells to simulate mosaicism in PGD specimens. They noted a shift in the $\log _{2}$ ratio when mosaicism reached $25 \%$ and confidently interpreted the presence of mosaicism when it was present at the 50\% level for chromosome gains. They did not present data for chromosome losses. Northrop et al. [14] combined cells, in graded proportions, from normal male and female cell lines to simulate mosaic blastocyst biopsies and then analyzed these mixed cell populations using a $262 \mathrm{~K}$ SNP microarray. Their results indicated that identification of monosomy X mosaicism (male cells) was detectable when $>25 \%$ of cells in the population were male. Scott et al. [22] mixed DNA samples extracted from cytogenetically normal and abnormal sources, without whole genome amplification, to determine the limits of mosaicism detection using a $44 \mathrm{~K}$ oligonucleotide array. Mosaicism as low as $10 \%$ for both gains and losses of whole chromosomes was detectable when data from dye-reversed replicates were combined but this detection limit rose to 20-30\% mosaicism in the absence of dye-reversed replicates. While high resolution arrays may detect lower levels of mosaicism, the primary purpose of array testing in the PGD setting is to identify whole chromosome aneuploidy without the additional uncertainty associated with the interpretation of the clinical significance of small copy number variants. Therefore, with this clinical objective in mind and with the technical limitations imposed by small sample sizes and the requirement for whole genome amplification, embryo mosaicism of less than approximately $25 \%$ will not likely be detectable.

After establishing threshold levels for mosaicism identification, these criteria were applied to classify embryos as euploid, non-mosaic aneuploid, or mosaic aneuploid. Follow-up FISH studies of our abnormal embryos were evaluated to assess the diagnostic accuracy of CMA testing. In a series of 47 embryos with follow-up data, $26 \mathrm{em}$ bryos were considered to be non-mosaic by CMA. While FISH confirmed whole chromosome aneuploidy in all of the embryos (100\%), 13 embryos in this group (50\%) were mosaic by FISH for at least one chromosome. Among the 
possible reasons for this observation is unequal distribution of mosaic cells between the inner cell mass (ICM) and TE or the effect of sample size. Recent studies of cells derived from TE and ICM and analyzed by CMAs by Johnson et al. [23] and Northrup et al. [14] do not support the preferential segregation of aneuploid cells to the TE. These studies are also consistent with an earlier report by Evsikov and Verlinsky using FISH [24]. Because more embryonic cells are available for reanalysis by FISH compared to the limited number of cells in a TE biopsy, it is likely that some embryos diagnosed as non-mosaic aneuploid by CMA will display mosaicism by FISH due to sampling error. This observation has been reported by Fragouli et al. [13] when they compared CGH data and FISH reanalysis results. Therefore, we reported an embryo as confirmed abnormal if at least one chromosome in an embryo identified by CMA was also identified as abnormal (non-mosaic or mosaic) by FISH. These criteria are similar to that utilized by Gutierrez-Mateo et al. [25] in their study of embryo reanalysis by FISH. Another consideration regarding mosaic embryos is that embryos can be aneuploid-aneuploid or aneuploid-euploid. While the clinical significance of low levels of mosaicism is uncertain, most likely aneuploid-aneuploid mosaic embryos (no euploid cells) will not lead to viable live births. In our study all mosaic embryos were aneuploid-euploid.

If there is an equal distribution of cells within the embryo, a large biopsy will inherently better reflect the total embryonic population and will be less susceptible to sample bias compared to a small biopsy. Of the $13 \mathrm{em}-$ bryos in the CMA non-mosaic category which by FISH follow-up were identified as mosaic, 10/13 (78\%) of these embryos had three or less cells in the biopsy. Among 21 embryos identified as mosaic by CMA, 20 (95\%) were confirmed as abnormal by FISH. Interestingly, one embryo in this group was non-mosaic by FISH. The single case with a discordant result had $7 \%$ of nuclei with an aneuploid FISH signal which was slightly below our threshold for scoring this embryo as abnormal. In this study we only attempted to confirm mosaicism by FISH for chromosomes identified by CMA to be abnormal. While it is possible that this approach underestimated the number of mosaic chromosomes present in embryos, this is not likely because in our preclinical validation studies we established high analytical sensitivity using CMA. No FISH follow-up studies were performed on embryos identified by CMA to be euploid because these embryos were generally not available for further study and because our preclinical validation experiments demonstrated high analytical specificity.

CMA testing of TE biopsies from unselected blastocyst populations demonstrates high levels of chromosomally abnormal embryos. In a recent randomized clinical trial, Yang et al. [16] reported aneuploidy in $56.6 \%$ of blastocysts from young, good prognosis patients. With aCGH(CMA) or CGH, Fragouli et al. [13] reported 42.3\% blastocyst euploidy, 30\% non-mosaic aneuploidy, and $32.4 \%$ mosaic aneuploidy. Using the mosaicism thresholds described above, we analyzed 551 day $5 / 6$ embryos by CMA. Of these, $52.5 \%$ were diagnosed to be euploid and $47.5 \%$ to be chromosomally abnormal. In the abnormal embryo population, $40.5 \%$ were non-mosaic aneuploid, $31.6 \%$ mosaic only, and $27.9 \%$ of embryos displayed nonmosaic and mosaic aneuploidy together. However, based on our FISH follow-up studies that showed that about one half of CMA diagnosed non-mosaic embryos were, in fact, mosaic because of skewed sampling, then the size of the mosaic class of embryos would be expected to be larger. When the same CMA data were analyzed by age group, younger women (25-34 years old), as expected, had the highest percent of euploid embryos (59.8\%) and older women (40-42 years old) had the lowest percent of euploid embryos (24\%). The ratio of non-mosaic aneuploid embryos to euploid embryos was 0.24 in the youngest age group and 1.67 in the oldest age group. This observation is consistent with a maternal age effect for meiotic nondisjunction. The ratio of mosaic only embryos to euploid embryos was relatively constant among the different age groups: $0.26,0.35$, and 0.33 for the $25-34,35-39$, and $40-$ 42 year old patients, respectively. While the number of embryos available for study for the 40-42 year old group was small and thus insufficient for statistical comparison, there does not appear to be striking differences between these groups for the mosaic only category. If larger studies confirm this observation, then mitotic non-disjunction leading to mosaicism would appear to be a random event unrelated to maternal age. With demonstrated maternal age effects on meiotic non-disjunction as well as egg cohort size, the practical significance of a steady rate of mitotic non-disjunction across age groups is that it can lead to a less complicated estimation of the number of normal blastocysts that can be expected given the number of fertilized eggs available and a women's maternal age.

This study confirms that mosaicism is common in day $5 / 6$ blastocysts and that CMA testing can identify a significant proportion of these embryos. FISH follow-up studies confirm that mosaicism suggested by CMA profiles is not likely to be due to technical artifacts of the arrays and thus the likelihood that normal embryos are misidentified and not transferred is reduced. Some abnormal mosaic embryos, however, may not be identified because the TE biopsy may not be representative of the embryo and it may yield only euploid cells. It is important, then, that laboratories perform pre-clinical mixing experiments to validate the performance characteristics of their array platform and to establish detection thresholds for mosaicism in order to reduce the chances of transferring abnormal embryos. Preliminary studies in 
our laboratory using next generation sequencing suggest that this approach can also be sensitive in the identification of blastocyst mosaicism.

\section{Conclusions}

In this study we report the data from reconstitution experiments using DNA mixed from normal and aneuploidy specimens to define the limits of detection of mosaicism using CMA as well as the accuracy of aneuploidy and mosaic aneuploidy identification in blastocyst TE samples. Our findings underscore the challenges in the identification of embryos with low levels of chromosomal mosaicism and the importance of obtaining sufficient cells in the TE specimen to minimize the effects of skewed biopsy sampling.

\section{Methods}

\section{Study design and materials}

Experimental and laboratory studies were performed on karyotypically defined human fibroblast cell lines and human IVF embryos donated to quality assurance follow up. Patients' CMA data was calculated based on analysis of 551 embryos obtained from 120 IVF cycles (103 patients total).

\section{Ovarian stimulation/IVF/TE biopsy}

All embryos analyzed in this study were obtained during IVF cycles following ovarian stimulation by standard protocols as administered by the patients' physicians. Intracytoplasmic sperm injection (ICSI) and day 3 assisted hatching procedures were routinely performed on all cases as required by our PGD protocol. The trophectoderm biopsy was done on day $5 / 6$ hatching blastocysts, with an average biopsy size of around four-five cells based on microscopic estimation using a laser on the constricted area of herniating TE. A modification of Gardner's blastocyst scoring system [26] was used for grading embryos that became hatching blastocysts on day 5 or day 6 . In short, embryos were rated based on three criteria: (i) the degree of expansion - 6.2 to 6.6 , (ii) the quality of inner cell mass - A to C, (iii) the quality of trophectoderm cells - X to Z. About 29\% of all hatching blastocysts were scored as AX, $\sim 25 \%$ were scored as $\mathrm{AY}, \sim 11 \%$ were score as $\mathrm{BX}, \sim 30 \%$ were scored as $\mathrm{BY}$ and $\sim 5 \%$ were scored as $\mathrm{AZ}, \mathrm{CX}, \mathrm{BZ}$.

\section{Reconstitution experiments}

DNAs from aneuploid and euploid cell lines as well as clinical samples were mixed in different ratios $(1: 7$ [12.5\%], 1:3 [25\%], 3:5 [37.5\%], 1:1 [50\%], 5:3 [62.5\%], $3: 1$ [75\%] and $7: 1$ [87.5\%]) for reconstitution experiments. For chromosomal gains, trisomy 13, trisomy 21 and euploid male cell lines (GM00526, GM02067B and GM05386B, respectively; (Coriell Cell Repositories, USA) were used. DNA from five cells of each cell line was amplified using the SurePlex kit (BlueGnome, UK), mixed, and assayed by array comparative genomic hybridization (aCGH; BlueGnome). Reconstitution experiments to obtain the individual $\log _{2}$ ratios for trisomy 13 and trisomy 21 were independently repeated at least three times. For chromosomal losses, amplified DNA from patient specimens that were identified by CMA and confirmed by FISH to have full monosomy 6 and monosomy 22 were used. The trophectoderm biopsy samples from these sources contained five cells. For all reconstitution experiments, $\log _{2}$ ratios were calculated to express deviation of CMA profiles at each level of sample mixing. We interpreted the presence of mosaicism when all hybridization signals for a chromosome were uniform and visually separated above or below the $0 \log _{2}$ ratio line; that is, when there was no scatter of individual hybridization signals or small groups of hybridization signals that closely approached or touched the $0 \log _{2}$ ratio line (see Additional files 3 and 4). For a diagram of a reconstitution experiment please see the Additional file 6.

\section{CMA (aCGH)}

The BlueGnome SurePlex and 24sure array kits were used for whole genome amplification (WGA) and subsequent labeling/hybridization of DNAs derived from fibroblast cells and TE specimens, following the manufacturer's protocol (BlueGnome, UK). Slides were scanned using PowerScanner (Tecan, Switzerland) and TIFF images were analyzed and interpreted using $\log _{2}$ ratios calculated by BlueFuse Multi software (BlueGnome, UK). Embryos identified to be aneuploid (non-mosaic or mosaic) by CMA from TE specimens were reanalyzed, when available, by FISH to confirm the array diagnosis. The BlueFuse Multi software algorithm for making euploid and aneuploid calls with 24sure arrays is based on the median $\log _{2}$ ratio of each chromosome. A chromosome gain will be called when the median $\log _{2}$ ratio reaches +0.225 with $51 \%$ confidence in the call but will achieve $100 \%$ confidence when the median ratio is $\geq+0.35$. A chromosome loss is called when the median $\log _{2}$ ratio is $<-0.375(51 \%$ confidence) and at $100 \%$ confidence when the median ratio is $\leq-0.6$ (BlueGnome; personal communication). In addition to software algorithms, there are interexperimental variables which also affect the ability to define the limits of detection of mosaicism. These include variability in array lots, biopsy size, and integrity of DNA in biopsied cells.

\section{Embryo follow-up studies}

Blastocysts used for quality assurance follow-up studies were abnormal embryos donated for this purpose following an informed consent process. Establishing a legal disposition for every embryo created by IVF is mandatory. 
The purpose and importance of embryo follow-up studies is presented by a genetic counselor at the first PGD consult and detailed in patient take-home PGD materials. After getting this information, each patient must sign a written consent for embryos disposition - embryos may be frozen, discarded or donated to research. Patients are aware these decisions are entirely voluntary and that they will not directly benefit from them. The embryo donation research consent includes permission to publish aggregate data. The Genetics and IVF Institute (GIVF) Institutional Review Board (IRB) is registered with the United States Department of Health and Human Services (HHS) and adheres to their guidelines for the protection of human subjects. The research described in this publication consisted of the analysis of existing data from donated specimens obtained by written informed consent for the purpose of continuous PGD quality assurance (QA) monitoring. It was determined by the Chairperson of the GIVF IRB that this study qualified as exempt research as promulgated by the U.S. Department of Health and Human Services because the materials already existed for QA purposes and the analysis of records presented no risk to patients.

For FISH studies, blastocysts were dissociated by first removing the zona pellucida with $1000 \mu \mathrm{s}$ bursts from a laser (Hamilton Thorne Biosciences, USA) or with treatment with $0.1 \%$ pronase. Embryos were then placed in $50 \mu \mathrm{l}$ of $\mathrm{Ca} / \mathrm{Mg}$-free medium with $5 \% \mathrm{HSA}$ [QA Medium with HEPES (Sage)/human serum albumin (100 $\mathrm{mg} / \mathrm{ml}$ )] at room temperature for 15 minutes. Embryos were transferred to a $2 \mu$ droplet of hypotonic solution (50\% Dulbecco's phosphate buffered saline) on a glass slide for approximately 2 minutes. To the hypotonic solution was added $15 \mu \mathrm{l}$ of lysis solution $(0.01 \mathrm{~N} \mathrm{HCl}$, $0.3 \%$ Tween 20). After approximately 10 minutes, most cells will have separated from each other with the cell membrane lysed. The slides were dried at room temperature or on a $37^{\circ} \mathrm{C}$ slide warmer. Cells were fixed for 5 minutes in $50 \mathrm{ml}$ of $2.5 \%$ buffered formalin phosphate solution (Dulbecco's phosphate buffered saline/10\% buffered formalin phosphate) before being rinsed in deionized water and dehydrated for 5 minutes each in a $70 \%$, $90 \%$, and $100 \%$ ethanol series. Slides were dried at room temperature. FISH probes (Abbott, USA) used in followup studies targeted chromosomes identified to be abnormal by CMA analysis. Because of reduced hybridization efficiencies with multiple cycles of FISH on the same cell, only 2-3 chromosomes were targeted in follow-up in embryos containing multiple chromosome aneuploidies identified by CMA. Probes and target DNA were co-denatured at $75^{\circ} \mathrm{C}$ for 5 minutes using a HYBrite instrument (Abbott) and then transferred to a humidified chamber at $37^{\circ} \mathrm{C}$ overnight. Following hybridization, slides were first washed for 2 minutes in $0.4 \mathrm{X}$ SSC (sodium chloride/sodium citrate) at $72-72^{\circ} \mathrm{C}$ and then in $2 \mathrm{XSSC} /$ $0.1 \%$ Igepal at room temperature for 1 minute. Slides were then rinsed at room temperature in $\mathrm{PN}$ buffer $(100 \mathrm{mM}$ $\mathrm{Na}_{2} \mathrm{HPO}_{4}$ / $50 \mathrm{mM} \mathrm{NaH} \mathrm{PO}_{4}$ / 0.1\% Igepal). Cells were counterstained with DAPI (Abbott, USA) and approximately 25-35 nuclei with good signal intensity were scored per embryo using a fluorescence microscope (Olympus) and a 60X oil immersion objective. The interpretation of interphase FISH data is subject to technical errors due to overlapping, split, weak, or faded signals. From our experience (unpublished data) and descriptions by others [27], we estimate there is an approximate $10 \%$ total error rate in FISH scoring when 2-3 probes are applied simultaneously. Therefore, we categorized embryos as nonmosaic aneuploid when $90 \%$ or more cells were scored as aneuploid and embryos as mosaic when $<90 \%$ of nuclei but $\geq 10 \%$ of nuclei had an aneuploidy for any single chromosome.

With an approximately $10 \%$ FISH error rate, it is possible that some abnormal embryos categorized by FISH to be mosaic could, in fact, have been non-mosaic when FISH results were near our upper threshold. In this instance, however, FISH would still have confirmed an abnormality in the embryo. It is also possible that some embryos with very low levels of mosaicism by FISH could have been euploid. In our follow-up experiments, however, very few embryos had FISH scores close to our lower threshold and thus this would not have materially affected the general conclusion of our study. Our CMA studies were validated only for whole chromosome aneuploidies and therefore, FISH follow-up data from embryos suggestive of containing segmental aneuploidies alone or in combination with other abnormalities were excluded.

\section{Additional files}

Additional file 1: Reconstitution Experiments - Chromosome Gains. Individual $\log _{2}$ ratios and average $\log _{2}$ ratios were determined for trisomy 13 and trisomy 21 samples at different levels of mosaicism.

Additional file 2: Reconstitution Experiments - Chromosome Losses. Individual $\log _{2}$ ratios and average $\log _{2}$ ratios were determined for monosomy 6 and monosomy 22 at different levels of mosaicism.

Additional file 3: Chromosomal microarray profiles of chromosomal aneuploidy for a trisomy (+13). Images (A) - (F) showed different levels of aneuploidy in the experimental samples: at $0,25,37.5,50,75$ and $100 \%$, respectively.

Additional file 4: Chromosomal microarray profiles of chromosomal aneuploidy for a monosomy (-6). Images (A) - (F) showed different levels of aneuploidy in the experimental samples: at $0,25,37.5,50,75$ and $100 \%$, respectively.

Additional file 5: Blind-test results of mosaic and non-mosaic samples.

Additional file 6: Diagram of a reconstitution experiment. The visual illustration of how reconstitution (mixing) experiments were performed in this study. 


\section{Abbreviations}

aCGH: array Comparative genomic hybridization; CMA: Chromosomal microarrays; ICSI: Intracytoplasmic sperm injection; IRB: Institutional review board; IVF: In Vitro fertilization; FISH: Fluorescence In situ hybridization; PGD: Preimplantation genetic diagnosis; TE: Trophectoderm.

\section{Competing interests}

The authors declare that they have no competing interests.

\section{Authors' contributions}

VN was involved in acquisition, analysis and interpretation of data, drafting and revising the article. EBM performed embryo spreading and FISH followup studies and helped with editing the paper. MES performed embryo spreading and FISH follow-up studies and helped with editing the paper. SLS took part in reconstitution experiments. KDT helped with acquisition of data. HJS substantially contributed to conception and design, critically revised the article and participated in the final approval of the version to be published. BDM substantially contributed to conception and design, critically revised the article and participated in the final approval of the version to be published. WSS helped to draft and revise the final version of the paper, and substantially contributed to the experimental design, and data interpretation. All authors read and approved the final manuscript.

\section{Acknowledgements}

The authors thank Dr. Donald Marazzo for critically reviewing this manuscript and for valuable suggestions.

Received: 14 November 2013 Accepted: 11 February 2014

Published: 28 February 2014

\section{References}

1. Hsu LY, Perlis TE: United States survey on chromosome mosaicism and pseudomosaicism in prenatal diagnosis. Prenat Diagn 1984, 4:97-130.

2. Wilson MG, Lin MS, Fujimoto A, Herbert W, Kaplan FM: Chromosome mosaicism in 6,000 amniocenteses. Am J Med Genet 1989, 32:506-513.

3. Grati FR, Grimi B, Frascoli G, Di Meco AM, Liuti R, Milani S, Trotta A, Dulcetti F, Grosso E, Miozzo M, Maggi F, Simoni G: Confirmation of mosaicism and uniparental disomy in amniocytes, after detection of mosaic chromosome abnormalities in chorionic villi. Eur J Hum Genet 2006, 14:282-288.

4. Ledbetter DH, Zachary JM, Simpson JL, Golbus MS, Pergament E, Jackson L, Mahoney MJ, Desnick RJ, Schulman J, Copeland KL, Verlinsky Y, Yang-Feng T, Schonberg SA, Babu A, Tharapel A, Dorfmann A, Lubs HA, Rhoads GG, Fowler SE, De La Cruz F: Cytogenetic results from the U.S. Collaborative Study on CVS. Prenat Diagn 1992, 12:317-345.

5. Wang $\mathrm{BB}$, Rubin $\mathrm{CH}$, Williams J 3rd: Mosaicism in chorionic villus sampling: an analysis of incidence and chromosomes involved in 2612 consecutive cases. Prenat Diagn 1993, 13:179-190.

6. Hahnemann JM, Vejerslev LO: Accuracy of cytogenetic findings on chorionic villus sampling (CVS)-diagnostic consequences of CVS mosaicism and non-mosaic discrepancy in centres contributing to EUCROMIC 1986-1992. Prenat Diagn 1997, 17:801-820.

7. van Echten-Arends J, Mastenbroek S, Sikkema-Raddatz B, Korevaar JC, Heineman MJ, van der Veen F, Repping S: Chromosomal mosaicism in human preimplantation embryos: a systematic review. Hum Reprod Update 2011, 17:620-627.

8. Mertzanidou A, Wilton L, Cheng J, Spits C, Vanneste E, Moreau Y, Vermeesch JR, Sermon K: Microarray analysis reveals abnormal chromosomal complements in over $70 \%$ of 14 normally developing human embryos. Hum Reprod 2013, 28:256-264.

9. Wells D, Alfarawati S, Fragouli E: Use of comprehensive chromosomal screening for embryo assessment: microarrays and CGH. Mol Hum Reprod 2008, 14:703-710.

10. Harper JC, Sengupta SB: Preimplantation genetic diagnosis: state of the art 2011. Hum Genet 2012, 131:175-186.

11. Menasha J, Levy B, Hirschhorn K, Kardon NB: Incidence and spectrum of chromosome abnormalities in spontaneous abortions: new insights from a 12-year study. Genet Med 2005, 7:251-263.

12. Hodes-Wertz B, Grifo J, Ghadir S, Kaplan B, Laskin CA, Glassner M, Munne S: Idiopathic recurrent miscarriage is caused mostly by aneuploid embryos. Fertil Steril 2012, 98:675-680.
13. Fragouli E, Alfarawati S, Daphnis DD, Goodall NN, Mania A, Griffiths T, Gordon A, Wells D: Cytogenetic analysis of human blastocysts with the use of FISH, CGH and aCGH: scientific data and technical evaluation. Hum Reprod 2011, 26:480. 490.

14. Northrop LE, Treff NR, Levy B, Scott RT Jr: SNP microarray-based 24 chromosome aneuploidy screening demonstrates that cleavage-stage FISH poorly predicts aneuploidy in embryos that develop to morphologically normal blastocysts. Mol Hum Reprod 2010, 16:590-600.

15. Liu J, Wang W, Sun X, Liu L, Jin H, Li M, Witz C, Williams D, Griffith J, Skorupski J, Haddad G, Gill J: DNA microarray reveals that high proportions of human blastocysts from women of advanced maternal age are aneuploid and mosaic. Biol Reprod 2012, 87:148.

16. Yang Z, Liu J, Collins GS, Salem SA, Liu X, Lyle SS, Peck AC, Sills ES, Salem RD: Selection of single blastocysts for fresh transfer via standard morphology assessment alone and with array CGH for good prognosis IVF patients: results from a randomized pilot study. Mol Cytogenet 2012, 5:24.

17. Shaffer LG, Beaudet AL, Brothman AR, Hirsch B, Levy B, Martin CL, Mascarello JT, Rao KW: Microarray analysis for constitutional cytogenetic abnormalities. Genet Med 2007, 9:654-662.

18. Vermeesch JR, Fiegler H, de Leeuw N, Szuhai K, Schoumans J, Ciccone R, Speleman F, Rauch A, Clayton-Smith J, Van Ravenswaaij C, Sanlaville D, Patsalis PC, Firth H, Devriendt K, Zuffardi O: Guidelines for molecular karyotyping in constitutional genetic diagnosis. Eur J Hum Genet 2007, 15:1105-1114.

19. Manning M, Hudgins L: Array-based technology and recommendations for utilization in medical genetics practice for detection of chromosomal abnormalities. Genet Med 2010, 12:742-745.

20. Lamb AN, Rosenfeld JA, Coppinger J, Dodge ET, Dabell MP, Torchia BS, Ravnan JB, Shaffer LG, Ballif BC: Defining the impact of maternal cell contamination on the interpretation of prenatal microarray analysis. Genet Med 2012, 14:914-921.

21. Mamas T, Gordon A, Brown A, Harper J, Sengupta S: Detection of aneuploidy by array comparative genomic hybridization using cell lines to mimic a mosaic trophectoderm biopsy. Fertil Steril 2012, 97:943-947.

22. Scott SA, Cohen N, Brandt T, Toruner G, Desnick RJ, Edelmann L: Detection of low-level mosaicism and placental mosaicism by oligonucleotide array comparative genomic hybridization. Genet Med 2010, 12:85-92.

23. Johnson DS, Cinnioglu C, Ross R, Filby A, Gemelos G, Hill M, Ryan A, Smotrich D, Rabinowitz M, Murray MJ: Comprehensive analysis of karyotypic mosaicism between trophectoderm and inner cell mass. Mol Hum Reprod 2010, 16:944-949.

24. Evsikov S, Verlinsky Y: Mosaicism in the inner cell mass of human blastocysts. Hum Reprod 1998, 13:3151-3155.

25. Gutierrez-Mateo C, Colls P, Sanchez-Garcia J, Escudero T, Prates R, Ketterson K, Wells D, Munne S: Validation of microarray comparative genomic hybridization for comprehensive chromosome analysis of embryos. Fertil Steril 2011, 95:953-958.

26. Gardner DK, Lane M, Stevens J, Schlenker T, Schoolcraft WB: Blastocyst score affects implantation and pregnancy outcome: towards a single blastocyst transfer. Fertil Steril 2000, 73:1155-1158.

27. Sandalinas M, Sadowy S, Alikani M, Calderon G, Cohen J, Munne S: Developmental ability of chromosomally abnormal human embryos to develop to the blastocyst stage. Hum Reprod 2001, 16:1954-1958.

doi:10.1186/1755-8166-7-18

Cite this article as: Novik et al:: The accuracy of chromosomal microarray testing for identification of embryonic mosaicism in human blastocysts. Molecular Cytogenetics 2014 7:18. 